\title{
CATEGORÍAS MIXTAS TRUNCADAS: LA NOMINALIZACIÓN DEFECTIVA EN LAS CLÁUSULAS RELATIVAS EXISTENCIALES MODALES
}

\author{
TRUNCATED MIXED CATEGORIES: DEFECTIVE \\ NOMINALIZATION IN MODAL EXISTENTIAL RELATIVE CLAUSES
}

\author{
ANA OJEA \\ Universidad de Oviedo, Asturias. España \\ aojea@uniovi.es
}

\section{RESUMEN}

En este artículo se explora la estructura sintáctica de las denominadas Relativas Existenciales Modales (REM), construcciones que tienen forma oracional pero interpretación y distribución nominal (p. ej. No tengo con quién hablar). Se propone analizarlas como categorías mixtas truncadas, es decir, categorías no uniformes que se componen de un dominio discursivo verbal (SC) conectado con un dominio flexivo nominal (SQ) por medio de una categoría funcional SNom, que funciona como recategorizador. Este tipo de categorías mixtas son compatibles con los presupuestos restrictivos del Programa Minimista chomskiano y poseen una potencialidad empírica que aquí se trata de demostrar. En concreto, un análisis en esta línea permite dar cuenta de manera explícita de las semejanzas y diferencias de las REM con otras construcciones estructuralmente relacionadas con ellas (como son las cláusulas de relativo sin antecedente, las interrogativas indirectas o los sintagmas nominales escuetos), y también de su comportamiento ante procesos que afectan a la periferia oracional. Se defiende, por tanto que un adecuado tratamiento de las categorías mixtas redunda en beneficio de la descripción gramatical y abre interesantes vías de comparación interlingüística.

Palabras clave: Relativas Existenciales Modales, categoría mixta, categoría truncada, nominalización, Programa Minimista.

* Este trabajo se inscribe dentro del proyecto Estructura informativa y estructura argumental: investigación de interfaz en la sintaxis comparada de las lenguas germánicas y románicas (ref. FFI201341509-P), financiado por el Ministerio de Economía y Competitividad de España. 


\begin{abstract}
This paper explores the syntactic structure of the so called Modal Existential Relative Clauses, a type of construction with sentential form but nominal interpretation and distribution (e.g. No tengo con quién hablar). They are analyzed here as truncated mixed categories, that is, non-uniform categories made up of a verbal discourse domain (CP) connected to a nominal inflectional domain (QP) through the functional category NomP, which acts as a categorizer. It is argued that mixed categories of this sort fit within the restrictive programmatic assumptions of the Minimalist Program and that integrating them in the model brings forward interesting empirical predictions. In particular, an analysis along these lines serves to provide an explicit account of the similarities and differences between Modal Existential Relative Clauses and some other constructions structurally related to them (such as antecedent-less relative clauses, indirect questions and bare NPs); besides, it allows to explain the peculiarities in the articulation of their left periphery. It is therefore claimed that an adequate treatment of mixed categories benefits grammatical description and opens interesting paths for cross-linguistic analyses.
\end{abstract}

Keywords: Modal Existential Relative Clauses, mixed category, truncated category, nominalization, Minimalist Program.

Recibido: 17.09.2015. Aceptado: 04.04.2016.

\title{
1. INTRODUCCIÓN
}

La teoría sintáctica tiene como uno de sus objetivos básicos explicitar las condiciones de linealidad y jerarquía con que las distintas categorías se agrupan en constituyentes. En el Programa Minimista, la versión más reciente del modelo generativista chomskiano, existe para ello una única operación denominada ensamble (merge) que forma objetos sintácticos binarios y endocéntricos a partir de las piezas extraídas del léxico.

Ahora bien, no todos los constructos sintácticos encajan directamente en esta rígida concepción estructural y a menudo se encuentran con constituyentes aparentemente exocéntricos, es decir, que combinan propiedades de más de una categoría. Tal es el caso de las cláusulas subordinadas con verbo en infinitivo de los ejemplos (1)-(3), que, por razones que se señalarán más adelante, se denominarán Relativas Existenciales Modales:

(1) No hay qué comer.

(2) Estoy buscando con quién/ con quien hablar.

(3) No tienen dónde/donde ir. 
Estas cláusulas señalan entidades y, por tanto, aunque su forma es oracional su distribución y su interpretación las equipara a un constituyente nominal. Nótese que los verbos que las introducen no admiten argumentos proposicionales y que las secuencias (1)-(3) son de hecho equivalentes a oraciones de relativo cuyo antecedente es indefinido e inespecífico:

(4) No hay ninguna cosa que comer.

(5) Estoy buscando a alguien con quien hablar.

(6) No tienen ningún lugar donde ir.

Tal vez por esto la tradición gramatical española tiende a agruparlas con las oraciones de relativo, considerándolas un tipo especial de relativa sin antecedente, esto es, una oración que, tras un proceso que no siempre se explicita, se proyecta como un sintagma nominal ${ }^{1}$. Su estructura sintáctica debería ser, pues, semejante a la de los ejemplos de (7):

(7) Quien llamó parecía preocupado.

El que llamó parecía preocupado.

Ahora bien, existe una diferencia crucial entre las relativas sin antecedente (RSA) como las de (7) y las relativas existenciales modales (REM): las segundas no están sujetas a la que se ha denominado restricción de coincidencia (matching condition) que caracteriza a las primeras, y que fuerza al sintagma relativo introductor a ser compatible simultáneamente con los requisitos estructurales del predicado de la cláusula subordinada y con los del predicado de la oración principal; así, la RSA en (8b) es agramatical porque un sintagma preposicional relativo encabezado por la preposición por es compatible con los rasgos de subcategorización del verbo preguntar pero no con los del verbo soñar:

(8) a. Soñé con quien me presentaste ayer.

b. *Soñé por quien me preguntaste ayer.

Por el contrario, el ejemplo de (2) sí es posible a pesar de que el verbo buscar tampoco puede subcategorizar un sintagma preposicional encabezado por la preposición con.

Si en su forma y su distribución las REM combinan propiedades oracionales y nominales, en su estructura interna también exhiben propiedades duales ya que

${ }^{1}$ Este sería, mutatis mutandis, el análisis defendido, entre otros, en Bello (1847), Ramsey (1894), Fernández Ramírez (1951), Alcina y Blecua (1975), Plann (1980), Hirschbühler and Rivero (1981), Suñer (1984), Brucart (1999), Hernanz (1999), Gómez Torrego (2008), RAE (2009) y Masullo (2011). 
el sintagma QU- que las introduce puede recibir acento prosódico y ortográfico. Esto las distingue de las relativas y las asemeja a las cláusulas interrogativas indirectas, algo que ha sido destacado por los distintos gramáticos que han abordado su tratamiento. Bello (1847: 1106), por ejemplo, caracteriza las REM como oraciones de relativo con antecedente no explícito que expresan una idea general de persona, cosa o lugar. Ahora bien, el mismo señala más adelante que en ellas "el relativo se hace interrogativo indirecto" y, por tanto, "a veces será arbitrario dar o no a la frase la enunciación interrogativa” (Bello, 1847: 1110). Por las mismas fechas, Ramsey (1894: 19.43) ya denomina al sintagma QU- que las introduce "seeming relative" y reconoce que "some scholars consider the interrogative use to be the original one".

Existen otras propiedades estructurales que también asemejan a las REM más a las cláusulas interrogativas que a las relativas. Una de las más significativas es que permiten la elipsis de todo el material que sigue al sintagma QU- en contextos de coordinación (sluicing):

(9) a. Necesito hablar con alguien pero no tengo con quién.

b. Querrían irse a dormir pero no tienen dónde.

Esta opción se da también en las interrogativas indirectas (10), pero no así en las relativas, tanto si tienen antecedente explícito (11a) como si no (11b):

(10) Necesito hablar con alguien pero no sé con quién.

(11) a. *Necesito hablar con alguien pero no he encontrado a nadie con quien.

b. *Necesitaba hablar con alguien pero no he encontrado a quien / al que.

Así pues, para que un análisis de las oraciones (1)-(3) sea adecuado habrá de dar cuenta de esta naturaleza híbrida que las relaciona tanto con las oraciones de relativo como con las interrogativas indirectas ${ }^{2}$. Deberán para ello existir mecanismos que generen estructuras que, como las REM, exhiben una superposición de propiedades sintácticas. Tales estructuras serán "categorías mixtas", es decir, categorías no uniformes que combinan propiedades de dos proyecciones diferentes. Estas categorías han sido objeto de estudio frecuente en modelos formales como la LFG y GPSG, pero no así en el marco en que se inscribe esta investigación, el

\footnotetext{
${ }^{2}$ Significativamente, mientras que en las gramáticas del español se ha tendido a un análisis de las REM que enfatiza su carácter nominal y su relación con las oraciones de relativo, en la bibliografía sobre las construcciones equivalentes a (1)-(3) en otras lenguas la tendencia mayoritaria es considerarlas un tipo especial de cláusula más cercana estructuralmente a las interrogativas indirectas. Este tipo de análisis, inicialmente propuesto por Pesetsky (1982), se encuentra también en Grosu (1987, 1994, 2004), Grosu y Landman (1998), Izvorski (1998), Pancheva-Izvorski (2000), Caponigro (2001, 2003, 2004) y Šimík (2011), entre otros. También Bartra (1990) las analiza para el catalán como interrogativas indirectas.
} 
Programa Minimista chomskiano, aunque, como se verá, es posible integrarlas en sus presupuestos programáticos.

En lo que sigue, se describirá primero las características estructurales de las REM para justificar después, en la sección 3, la propuesta de analizarlas como categorías mixtas. En concreto, se propondrá que son categorías mixtas truncadas, es decir, cláusulas nominalizadas que no alcanzan el nivel discursivo y que este truncamiento es, de hecho, el que las diferencia de categorías mixtas plenas como son las RSA. La sección 4 explora las predicciones empíricas del análisis que se ofrece, sobre todo en lo que se refiere a la ordenación de la periferia izquierda oracional; y en la sección 5 se presentan las conclusiones.

\section{PROPIEDADES ESTRUCTURALES DE LAS RELATIVAS EXISTENCIALES MODALES EN ESPAÑOL}

La etiqueta que se utiliza aquí, Relativas Existenciales Modales, está basada en la acuñada por Grosu (2004) y Šimík (2011) para estas construcciones ${ }^{3}$. Ya se ha señalado que son relativas en tanto que funcionalmente equivalentes a una oración de relativo en infinitivo con antecedente indefinido e inespecífico (vid. (4)-(6)). Además, como Grosu (2004) y Šimík (2011) argumentan, son existenciales porque aparecen como complemento de verbos que, o bien señalan esa existencia (haber, tener), o permiten que esa existencia se infiera (buscar, encontrar... $)^{4}$. Finalmente, son modales porque tienen una interpretación modal de posibilidad (vid. en este sentido, Bello (1847: 650), Fernández Ramírez (1951: 375-9), Alcina y Blecua (1975: 1042), Demonte (1977: 96-100), Hernanz, (1999: 2292) y Masullo (2011: 317), entre otros).

Šimík (2011) constata en su monografía que estas oraciones están presentes en la mayor parte de las lenguas europeas, con la excepción de las germánicas, donde sólo el Yiddish y un dialecto neoyorkino del inglés parecen permitirlas (vid. también Caponigro (2003), para una lista exhaustiva de las lenguas que permiten REM).

En todas ellas las REM son oraciones modalizadas con verbo en infinitivo (o, en su defecto, en subjuntivo), se interpretan como constituyentes nominales indefinidos de lectura inespecífica, tienden a aparecer en configuraciones de polaridad marcada y están introducidas por sintagmas interrogativos.

Las tres primeras características se siguen del hecho de que las REM funcionan en general como complemento de verbos creadores de contextos opacos o inten-

\footnotetext{
${ }^{3}$ En concreto, Grosu (2004) y Šimík (2011) las denominan Cláusulas Existenciales Modales (Modal Existential Clauses).

${ }^{4}$ Tanto Grosu (2004) como Šimík (2011) ofrecen una lista exhaustiva de los verbos que pueden introducir REM en las distintas lenguas.
} 
sionales (vid. Demonte 1977; Táboas 1995), lo cual implica: a) que la existencia del referente que señala la REM quedará en suspenso (de ahí su lectura inespecífica) y b) que el verbo de la REM estará en modo no-indicativo, es decir, en infinitivo o subjuntivo (de ahí su lectura modal). En concreto, el subjuntivo será posible sólo cuando el elemento QU- funcione como sujeto de la REM, ya que en español este modo está sometido a la denominada "restricción de referencia disjunta" que impide que el sujeto de la oración principal y el de la subordinada en subjuntivo sean correferenciales ${ }^{5}$ :

(12) Busco $_{1 \text { pers }} /$ No tengo $_{{ }_{\text {pers }}} /$ No encuentro ${ }_{{ }_{\text {pers }}}$ con quién ir $\left(/ *^{*}\right.$ vaya $\left._{*_{1 \text { pers }}}\right)$.

(13) Busco $_{1 \text { pers }} /$ No tengo ${ }_{1 \text { pers }} /$ No encuentro ${ }_{1 \text { pers }}$ quien me ayude ${ }_{3 \text { pers }}\left({ }^{*}\right.$ ayudar $)$.

Respecto a la preferencia por entornos de polaridad marcada, ésta es una tendencia en las lenguas que poseen la construcción, pero no un requisito. En cualquier caso, nos encontramos aquí una vez más con una propiedad que viene determinada por la necesidad de que la REM aparezca en un contexto intensional, lo cual conlleva que se inserte en configuraciones que favorecen este tipo de contextos; esto incluye no solo oraciones de polaridad marcada como en (14) y (15), sino también oraciones de lectura genérica $(16)$ o condicional $(17)^{6}$ :

(14) No tengo dónde ir.

(15) ¿Tienes dónde ir?

(16) Siempre hay por quién vivir, por qué luchar.

(17) Si viniera Juan, ya tendríamos con quién terminar la tarea.

Finalmente, el punto más controvertido en la descripción gramatical de la construcción es, probablemente, la naturaleza del sintagma introductor. En los trabajos sobre el español, la mayor parte de los estudiosos consideran que el elemento QU- introductor es un operador relativo que opcionalmente puede ser tónico. En esta línea, Masullo (2011: 316) argumenta que el acento gráfico es simplemente una convención en la que se ha privilegiado el hecho de que este sintagma constituye el núcleo prosódico del enunciado. En términos semejantes se expresa la gramática de la Real Academia, que solamente señala que los relativos aquí se pronuncian unas veces de forma tónica y otras de forma átona (vid. RAE, 2009: 2024). Por su parte, Gómez Torrego (2008: 133), basándose en datos de

5 Sobre la restricción de referencia disjunta del subjuntivo ver, entre otros, Picallo (1984) y Kempchinsky (1986, 1990). Nótese que en las REM con subjuntivo el sintagma QU- tiende a no acentuarse, es decir, se identifica más con una forma relativa que en los casos en que este sintagma no es sujeto y aparece con preposición.

${ }^{6}$ Para una descripción exhaustiva de las configuraciones que inducen contextos intensionales véase, entre otros, Quer (2001) y RAE (2009: 1139 y ss). 
frecuencia de uso, señala que el introductor es relativo y aparece acentuado en función de su categoría, obligatoriamente si es preposicional y opcionalmente si no lo es.

Hay incluso quien como Plann (1980: 154) sostiene que el carácter tónico o átono del elemento introductor no es una variante libre, sino que se deriva de propiedades semánticas (y pragmáticas) distintas. Según esta autora, cuando el introductor es átono se presupone un antecedente indefinido e inespecífico:

(18) a. No había con que pulir el diamante (i.e. nada con que pulirlo).

b. No encuentro con que arreglar el techo (i.e. nada con que arreglarlo).

Sin embargo, si fuera acentuado, se referiría a algo específico. Por ejemplo, con qué arreglar el techo en (19) señalaría un instrumento específico para esa finalidad:

(19) No encuentro con qué arreglar el techo (i.e. un escayolador).

Para Plann (1980), por tanto, si el contexto no lo deja claro, ambas formas, la acentuada y la no acentuada, son posibles; sin embargo, el grado de especificación del contexto podría también determinar la obligatoriedad de una de las opciones:

(20) El miope al que se le rompen sus únicas gafas, no tiene con qué/*?que leer.

Los juicios de gramaticalidad de los hablantes nativos consultados (todos ellos hablantes de la variante castellana del español) no coinciden, en este sentido, con los expresados por Plann (1980), y en general el antecedente de la REM se entiende como inespecífico independientemente de que la forma introductora sea o no tónica. Lo que sí es innegable es que existe una gran indefinición a la hora de acentuar el elemento QU-. Por ejemplo, en (21a), oración tomada de Bello (1847), el introductor que aparece sin acento, mientras que, en la misma oración, Ramsey (1894) lo acentúa (la observación se debe a Plann, 1980: 152):

(21) a. No tengo que ponerme.

b. No tengo qué ponerme.

En la misma línea, el ejemplo (22a) aparece en RAE (2009: 2024) sin acento, mientras que el de (22b), muy semejante, es utilizado en la misma obra (RAE, 2009: 3339) con acento:

(22) a. No hay con quien hablar.

b. No hay de quién fiarse. 
Teniendo en cuenta que en español el inventario de los sintagmas relativos y el de los interrogativos coincide parcialmente y que, cuando lo hace, la única diferencia formal entre ambos es su carácter átono o tónico, es complicado determinar cuál es la naturaleza exacta del elemento introductor. Tres son las opciones posibles: a) siempre es un operador relativo que puede ser tónico o átono, b) es un operador relativo que puede sustituirse opcionalmente por uno interrogativo o c) es un operador interrogativo que se sustituye opcionalmente por uno relativo. De ellas, la comparación interlingüística parece favorecer esta última, ya que la inmensa mayoría de las lenguas donde son posibles las REM y donde interrogativos y relativos no son homófonos, el operador que las introduce es de tipo interrogativo (vid. Šimík, 2011). Significativamente, además, ni el complementador que, ni ningún relativo que carezca de homófono interrogativo en español puede introducir estas cláusulas:

(23) *Buscan que comer.

(24) *Busco con el cual pulir el diamante.

(25) *Busco con cuya ayuda pulir el diamante.

Se asume, por tanto, que, contrariamente a lo que a menudo se defiende, las REM están introducidas por sintagmas interrogativos y que, por una cuestión de asimilación con las oraciones de relativo sin antecedente, éstos pueden aparecer opcionalmente sin acento.

Así pues, las REM tienen un estatus intermedio entre una relativa sin antecedente y una interrogativa, ya que se interpretan como entidades (indefinidas e inespecíficas) al igual que las primeras, y comparten con las segundas el sintagma introductor y el no estar reguladas por el requisito de coincidencia (cf. 2). A continuación se presenta un análisis formal bajo los presupuestos del Programa Minimista que da cuenta de esta naturaleza híbrida y de las propiedades estructurales que de ella se siguen.

\section{LAS RELATIVAS EXISTENCIALES MODALES COMO CATEGORÍAS MIXTAS TRUNCADAS}

Las REM, por las propiedades que se acaban de señalar, constituyen un claro ejemplo de categoría mixta (mixed category), es decir, de categoría que combina uniformemente propiedades de dos proyecciones léxicas distintas. En el Programa Minimista este tipo de proyecciones han recibido muy escasa atención, a pesar de que, como han demostrado Panagiotidis y Grohmann (2005), su formalización puede encajar perfectamente con los presupuestos teóricos de este paradigma. 
Según Panagiotidis y Grohmann (2005), dos son los requisitos básicos de una categoría mixta:

a) Debe existir un recategorizador (SWITCH) entendido como una categoría funcional que relaciona dos categorías léxicas uniformes. Esta categoría tiene una especificación de rasgos dual (verbal y nominal) que será la que permita que se obtengan categorías también de naturaleza dual. En la práctica, el recategorizador es un nominalizador, un SNom que selecciona una categoría verbal y la convierte en nominal, pues no existe evidencia de categorías mixtas en las cuales el proceso se dé a la inversa (i.e. en que una proyección nominal se convierta en verbal). En anteriores trabajos (Ojea, 2011; 2013) se ha defendido la necesidad, además, de que el recategorizador SNom sea convenientemente activado, ya que la nominalización no es un proceso irrestricto que pueda afectar a cualquier categoría. En este sentido el rasgo nominal de SNom puede entenderse como interpretable pero no validado $([i \mu \mathrm{N}])$, y debe existir, por tanto, algún mecanismo de validación para que la categoría se active ${ }^{7}$.

b) Las categorías relacionadas por el recategorizador tienen que constituir un dominio prolífico (prolific domain; cf. Grohmann (2003: 74)), es decir, tienen que ser dos subestructuras susceptibles de ser interpretadas en las interficies relevantes. Tres son los dominios prolíficos posibles en la oración: el discursivo (que media la relación entre la proposición y el discurso: el Sintagma Complementante, convenientemente articulado), el flexivo (que estructura la información gramatical en torno a la cual se articula la oración, y que inaugura el Sintagma Tiempo) y el temático (donde se establece la relación entre el predicado y sus argumentos: las dos proyecciones verbales Sv y SV):

(26)

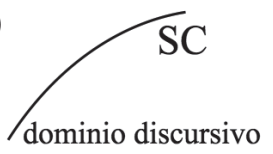

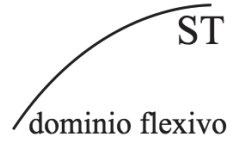

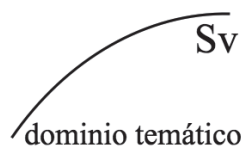

A menudo se asume que estos tres dominios se encuentran también en el ámbito de las categorías nominales (cf. Grohmann y Haegeman, 2002; Grohmann y Panagiotidis, 2005; Ticio 2003, 2006). Habría, por tanto, un dominio discursivo, que relaciona el sintagma nominal con la entidad extralingüística y capacita al SN para funcionar como argumento (SDet, donde se generan los determinantes fuertes en el sentido de Milsark, 1974); un dominio flexivo, que articula las rela-

${ }^{7}$ Adoptamos aquí la propuesta de Pesestsky y Torrego (2007), que desvincula la validación de un rasgo de su caracterización como [ \pm interpretable]. 
ciones gramaticales del nombre con sus modificadores (SQ, donde se proyectan inicialmente los cuantificadores); y, finalmente, un dominio temático, donde se hallaría el nombre con sus argumentos $(\mathrm{SN})$ :

(27)

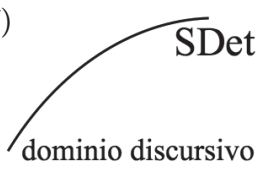

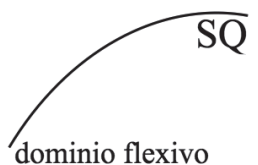

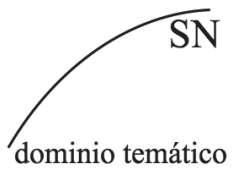

Con estos presupuestos, Panagiotidis y Grohmann (2005) abordan la caracterización de las cláusulas de gerundio en inglés como construcciones en las cuales un dominio temático verbal (SV) o un dominio flexivo verbal (ST) terminan proyectando un dominio discursivo nominal (SDet). Y en Ojea $(2011,2013)$ se defiende que un análisis del mismo tipo es el más adecuado para abordar la explicación de las oraciones de relativo sin antecedente. En concreto se defiende que todas las RSA en español pueden entenderse como categorías mixtas en las que un dominio discursivo verbal (i.e. una cláusula de relativo) se convierte en un dominio discursivo nominal (SDet) por la mediación del recategorizador SNom

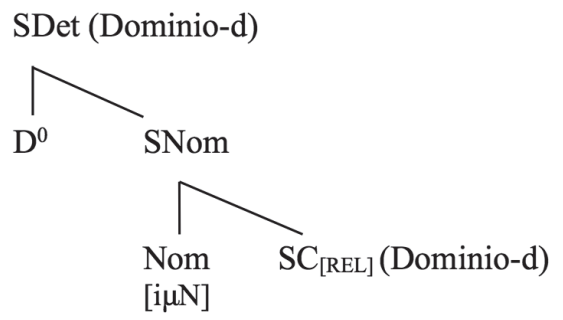

Las RSA se conciben, por tanto, como cláusulas que se recategorizan en SDet tras activarse SNom mediante el desplazamiento de un SDet relativo, previamente atraído al especificador de $\mathrm{SC}_{[\mathrm{REL}]}$, a su posición de especificador. En español ese operador relativo puede ser léxico o no-léxico; cuando es léxico podrá dotar a SNom de todos los rasgos semánticos y formales necesarios para la correcta interpretación de la categoría resultante. Este es el caso de las oraciones de relativo libres como (29), cuya derivación sería, en los detalles relevantes, como en (30):

${ }^{8}$ Nótese que la estructura de (28) es equivalente a la que tradicionalmente se ha defendido para las cláusulas de relativo con antecedente (cf. Ross (1967), Partee (1976) y Smits (1989), entre otros); la diferencia estructural entre las relativas con antecedente y las RSA se reduciría, por tanto, a que en las segundas habría un nominalizador SNom en lugar de la categoría léxica SN. 
(29) Quienes vinieron quedaron satisfechos.

(30) $\left[_{\text {SDet }}\left[{ }_{S \mathrm{Nom}}\right.\right.$ quienes $\operatorname{Nom}_{[\mathrm{i \mu N}]}\left[{ }_{S C}\right.$ quienes $[$ ST quienes vinieron $\left.\left.\left.]\right]\right]\right]$ quedaron satisfechos.

Por el contrario, cuando el operador relativo es no-léxico (lo cual, en español, siempre fuerza la aparición del complementador que en la relativa), el SDet resultante sólo será un objeto sintáctico válido si proyecta un determinante que dote a la proyección de los rasgos semánticos de los que ese operador nulo carece. Tal es la situación que se encuentra en las que se han denominado oraciones de relativo semilibres, como (31), y en las encabezadas por un relativo inespecífico compuesto de -quiera, como (33) (veáse Ojea (2013) para una justificación detallada de estos análisis):

(31) Los que vinieron quedaron satisfechos.

(32) $\left[_{\mathrm{SDet}} \operatorname{Los}\left[_{\mathrm{SNom}} O \mathrm{P}_{\mathrm{REL}} \operatorname{Nom}_{[\mathrm{i \mu N}]}\left[_{\mathrm{SC}} \Theta \mathrm{p}_{\mathrm{REE}}\right.\right.\right.$ que $\left[_{S T} \Theta \mathrm{p}_{\mathrm{REt}}\right.$ vinieron]]]] quedaron satisfechos.

(33) Quienquiera que venga quedará satisfecho.

(34) $\left[_{S D e t}\right.$ Quienquiera [SNom $O p_{\mathrm{REL}} \operatorname{Nom}_{[\mathrm{i \mu N}]}\left[_{\mathrm{SC}} O \mathrm{p}_{\mathrm{REE}}\right.$ que $\left[_{\mathrm{ST}} \Theta \mathrm{p}_{\mathrm{REE}}\right.$ venga]]]] quedará satisfecho.

Así pues, los distintos tipos de RSA en español pueden explicarse unitariamente como categorías mixtas obtenidas a partir de una cláusula de relativo. El SDet resultante es una expresión referencial que puede funcionar como argumento de cualquier predicado siempre y cuando cumpla con los requisitos de selección de éste; ésta es la base de la restricción de coincidencia (matching condition) que, como se señaló más arriba, constituye uno de los rasgos distintivos de las RSA.

Nuestra propuesta aquí es que la caracterización sintáctica de las REM puede abordarse en la misma línea: se estaría también ante categorías mixtas obtenidas, en este caso, a partir de una cláusula interrogativa con un operador-cuantificador referencial, es decir, el tipo de operador de las cláusulas interrogativas. Esta cláusula, en modo no-indicativo por la naturaleza intensional de los predicados que la seleccionan, se ensambla con la categoría funcional SNom y se proyecta eventualmente como una categoría nominal. Ahora bien, de la misma manera que el complemento de SNom puede ser un dominio prolífico de cualquier extensión (SC, ST o Sv), es de suponer que otro tanto ocurra con la categoría resultante, esto es, que pueda ser SDet, SQ o SN. O, lo que viene a implicar lo mismo, de la misma manera que existen categorías uniformes truncadas (i.e. cláusulas que solo se proyectan hasta ST, o proyecciones nominales que solo se proyectan hasta SQ), es esperable que existan categorías mixtas truncadas. Y este es, precisamente, el caso de las REM y lo que las convierte en construcciones marcadas y, por tanto, menos productivas interlingüísticamente que las RSA. En las REM el recategorizador SNom conecta un dominio discursivo con uno flexivo, es decir, convierte 
la cláusula (SC) en una categoría nominal truncada que sólo se proyecta hasta el nivel de SQ?:

(35) SQ (Dominio-f)

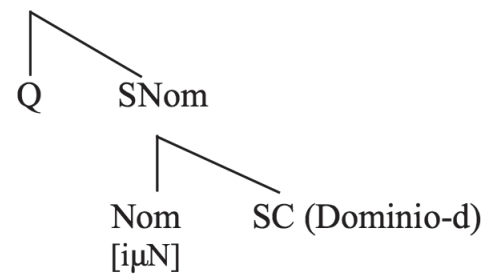

La estructura truncada de (35) explica, por una parte, que las REM no estén sujetas a la restricción de coincidencia que regula las RSA. El núcleo de SNom se valida aquí también mediante un operador QU- en el especificador de SNom. Pero puesto que ahora SNom no recategoriza la cláusula hasta SDet, ya no es necesario que el operador se restrinja a esta categoría; es posible, por tanto, encontrar sintagmas preposicionales encabezando la proyección aun cuando ésta funcione como objeto de un verbo transitivo:

(36) No tiene $\left[{ }_{\mathrm{SQ}}\left[{ }_{\mathrm{SNom}}\right.\right.$ de quién ${ }_{[\mathrm{i \mu N}]}\left[_{\mathrm{SC}}\right.$ te quiérn $\left[{ }_{\mathrm{ST}}\right.$ fiarse te quiérn $\left.\left.\left.]\right]\right]\right]$.

Por otra parte, puesto que la categoría resultante es SQ se predice que las REM tendrán una lectura de cuantificación existencial (cf. Pesetsky, 1982; Grosu, 2004; Šimík, 2011). Y también que estarán restringidas a posición postverbal, ya que la posición de sujeto preverbal en español sólo puede estar ocupada por una categoría plena (i.e. el dominio discursivo nominal DP). Es por ello que una REM nunca podrá ser afectada por la pasivización (cf. 37), de la misma manera que tampoco lo serán los sintagmas nominales escuetos, entendidos éstos igualmente como categorías (uniformes) truncadas que sólo se proyectan hasta SQ (cf. 38):

(37) a. Finalmente encontraron $[\mathrm{sQ}$ con quién hablar ].

b. ${ }^{*}{ }_{\mathrm{SQ}}$ Con quién hablar ] fue finalmente encontrado.

(38) a. Ese equipo restaura ${ }_{\mathrm{SQ}}$ capillas ].

b. ${ }^{*}\left[_{\mathrm{SQ}}\right.$ Capillas $]$ fueron restauradas por equipos especializados.

${ }^{9}$ Dada la relación estructural de las RSA con las REM, y puesto que esta última es la construcción marcada, es ciertamente esperable que los cuantificadores (átonos) de las primeras se utilicen, por un proceso de generalización, en las segundas; de ahí la fluctuación a la hora de acentuar las formas QU- en las REM. 
Un análisis de las REM como categorías mixtas truncadas permite, por tanto, dar cuenta de manera explícita tanto de su distribución como de sus principales propiedades estructurales y semánticas. Analizamos a continuación el comportamiento de las REM en procesos que implican la reordenación de la periferia izquierda (i.e. topicalización o focalización), ya que este comportamiento constituye una interesante evidencia en favor de nuestra propuesta.

\section{ESTRUCTURA SINTÁCTICA Y ESTRUCTURA INFORMATIVA EN LAS REM}

Hasta aquí se ha defendido un análisis de las REM que las relaciona directamente con las RSA en tanto que categorías mixtas obtenidas a partir de un constituyente oracional, y que explica las diferencias entre ambas en función de la naturaleza truncada de las primeras. Se espera, por tanto, que en procesos que afectan a la periferia de la cláusula base ambas construcciones se comporten de manera similar, mientras que en procesos que implican extracción desde la categoría resultante (SDet en el caso de las RSA y SQ en el de las REM) exhiban diferencias significativas.

Respecto del primer punto, las REM comparten con las RSA el hecho de que son categorías que se constituyen a partir de un dominio discursivo (SC); ambas implican también el movimiento de un operador al especificador de $\mathrm{SC}$-interrogativo en el caso de las primeras, relativo en el de las segundas- como paso previo a la activación del nominalizador SNom. En las categorías uniformes en las que tiene lugar este mismo proceso (i.e. las cláusulas interrogativas indirectas o las cláusulas de relativo con antecedente), el operador en SC es compatible con la existencia de sintagmas topicalizados que comparten con él la periferia oracional. Tal es el caso del sintagma las maletas en los ejemplos siguientes:

(39) Me pregunto por qué las maletas no las dejaron en mi habitación. (cf. Me pregunto por qué no dejaron las maletas en mi habitación).

(40) No me dijeron la razón por la cual las maletas no las habían dejado en mi habitación. (cf. No me dijeron la razón por la cual no habían dejado las maletas en mi habitación).

Esto ha llevado a que, desde el trabajo seminal de Rizzi (1997), se haya defendido que SC posee una estructura articulada con una serie de categorías opcionales donde se sitúan los distintos tópicos y focos oracionales ${ }^{10}$.

${ }^{10}$ Por razones de espacio no se entró a considerar aquí cuáles son las proyecciones que articulan SC ni cómo se ordenan entre sí, tema este que ha recibido mucha atención en la bibliografía reciente (véase, por ejemplo, Frascarelli (2012) y las referencias que allí se citan). 
Ahora bien, frente a lo que ocurre en (39) y (40), topicalizar un constituyente dentro de una REM resulta, cuando menos, forzado:

(41) *No tengo dónde las maletas dejarlas.

(cf. No tengo dónde dejar las maletas).

(42) *?No encuentro quién esa tarea me la haga de buen grado.

(cf. No encuentro quién me haga esa tarea de buen grado.

La agramaticalidad de (41) y (42) no pueden deberse al modo no-indicativo de la REM, ya que, si bien es cierto que el infinitivo es incompatible con los procesos de topicalización, el subjuntivo no los impide en la mayor parte de los contextos ${ }^{11}$ :

(43) Negaron que los libros los hubieran cambiado de sitio.

Por otra parte, se encuentran los mismos problemas en las RSA (también categorías mixtas), incluso cuando su modo es indicativo:

(44) *? Te presento a quien/al que esa tarea te la hará de muy buena gana.

(cf. Te presento a quien/al que te hará esa tarea de muy buena gana).

Así pues, la dificultad para topicalizar constituyentes en las REM y en las RSA tiene que deberse a su naturaleza como categorías mixtas y resulta esperable desde el momento en que SC no es aquí un objeto sintáctico pleno (i.e. conectado con el discurso y capaz, por tanto, de reorganizarse por razones discursivas), sino parte de una construcción sintáctica compleja.

En lo que se refiere a la categoría resultante, hemos defendido que en el caso de las REM se obtiene un SQ, categoría truncada que no constituye una fase a efectos computacionales. Será accesible, por tanto, a cualquier operación que se efectúe en un ciclo superior, como podemos comprobar tanto en procesos de topicalización (ejemplos (45) y (46)) como de focalización (ejemplos (47) y (48)):

(45) Las maletas no tengo dónde dejarlas.

(cf. No tengo dónde dejar las maletas).

(46) Esa tarea no encuentro quién me la haga de buen grado.

(cf. No encuentro quién me haga esa tarea de buen grado).

(47) ¿¿De qué no tienes con quién hablar?

(cf. No tengo con quién hablar de política).

${ }^{11}$ La imposibilidad de topicalizar (o focalizar) constituyentes en las oraciones de infinitivo se ha explicado en términos de defectividad estructural del dominio discursivo en estas cláusulas (vid. Hernanz, 2011), o de la opacidad de su ST para heredar rasgos discursivos (vid. Jimenez-Fernández y Miyagawa, 2014). 
(48) ¿Para quién estás buscando qué comprar?

(cf. Estoy buscando qué comprar para mi madre).

Se predice así, correctamente, que en este aspecto diferirán de las RSA, cláusulas mixtas no truncadas (SDet) que constituyen, por tanto, contextos opacos a efectos de extracción ${ }^{12}$ :

(49) * Esa tarea conozco a quien/al que te la podría hacer de buen grado.

(cf. Conozco a quien/al que podría hacerte esa tarea de buen grado).

(50) * ¿De qué has conocido a quien/al que ayer hablaba con María?

(cf. He conocido a quien /al que ayer hablaba de política con María).

Así pues el comportamiento de las REM ante procesos de topicalización y focalización refleja de manera significativa la especial naturaleza de estas construcciones en tanto que proyecciones defectivas tanto en el nivel oracional (i.e. no permiten la reorganización de sus constituyentes por cuestiones discursivas ya que no funcionan como cláusulas en las interficies), como en el nivel nominal resultante (i.e. no constituyen una fase a efectos computacionales debido a su truncamiento estructural).

\section{CONCLUSIONES}

En este trabajo se ha abordado la descripción y el análisis de las Relativas Existenciales Modales en tanto que categorías que combinan propiedades oracionales y nominales. Se han tratado como categorías mixtas (truncadas) en la línea de lo defendido por Panagiotidis y Grohmann (2005) para las cláusulas de gerundio en inglés. Las categorías mixtas así entendidas combinan un dominio prolífico verbal con un dominio prolífico nominal, conectados ambos por una categoría funcional que actúa como recategorizador. Su estructura y funcionamiento se sigue de los principios generales de la gramática, lo cual las convierte en categorías marcadas (frente a las categorías uniformes), pero no excepcionales.

Se demostró que caracterizar una construcción como categoría mixta permite no sólo ahondar en sus propiedades de una manera explícita, sino también explicar las semejanzas y diferencias que esa construcción tiene con: a) la categoría que sirve como base, b) la categoría que se obtiene tras la activación del nominalizador y c) otras categorías mixtas. En esta línea se pudo dar cuenta de las semejanzas y

${ }^{12}$ Otro tanto ocurre con construcciones relacionadas estructuralmente con las REM, tales como las relativas con antecedente o las interrogativas indirectas: ninguna de ellas es una categoría truncada y por tanto, completada la fase, son opacas para movimientos posteriores:

i) * ¿De qué no tienes a nadie con quien hablar?

ii) ${ }^{*}$ ¿De qué te preguntas si hablé ayer con María? 
diferencias de las Relativas Existenciales Modales con las cláusulas interrogativas indirectas, con los sintagmas nominales escuetos (que, al igual que ellas, sólo se proyectan hasta SQ) y con las relativas sin antecedente.

Como es bien sabido, por otra parte, la variación lingüística está en gran medida determinada por las categorías funcionales presentes en las distintas lenguas naturales, y por los rasgos formales que las definen. Creemos que incorporar una categoría como SNom a ese inventario permite el análisis explícito tanto del tipo de categorías mixtas que cada lengua posee como de los mecanismos que las hacen posibles. Se abren así interesantes vías de comparación entre construcciones y entre las lenguas en que estas construcciones aparecen, con el consiguiente beneficio para la teoría lingüística.

\section{REFERENCIAS}

Alcina Franch, Juan y Blecua, José Manuel. (1975). Gramática española. Barcelona, España: Ariel.

Bartra, Anna. (1990). Sobre unes frases relatives sense antecedente. Caplletra, 8 , 131-148.

Bello, Andrés. (1847). Gramática de la lengua castellana destinada al uso de los americanos. Tenerife, España: Aula de Cultura de Tenerife, 1981.

Brucart, José $M^{a}$. (1999). La estructura del sintagma nominal: las oraciones de relativo. En Ignacio Bosque y Violeta Demonte (Eds.), Gramática descriptiva de la lengua española (pp. 395-522). Madrid, España: Espasa Calpe.

Caponigro, Ivano. (2001). On the semantics of indefinite free relatives. En Marjo van Koppen, Joanna Sio y Mark de Vos (Eds.), Proceedings of ConSOLE 10 (pp. 49-62). Leiden, Holanda: SOLE.

Caponigro, Ivano. (2003). On the semantics of free relatives and wh-words crosslinguistically. Tesis doctoral. California, Estados Unidos: UCLA.

Caponigro, Ivano. (2004). The semantic contribution of wh-words and type shifts: Evidence from free relatives crosslinguistically. En Robert B. Young (Ed.), SALT 14: Proceedings from the 14th Conference on Semantics and Linguistic Theory (pp. 38-55). Ithaca, NY, Estados Unidos: CLC Publications.

Demonte, Violeta. (1977). La subordinación sustantiva. Madrid, España: Cátedra. Fernández Ramírez, Salvador. (1951). Gramática española. 4. El verbo y la oración. Madrid, España: Arco Libros, 1986.

Frascarelli, Mara. (2012). The interpretation of discourse categories: Cartography for a crash-proof syntax. En Valentina Bianchi y Cristiano Chesi (Eds.), Enjoy Linguistics! Papers offered to Luigi Rizzi on the occasion of his 60th birthday (pp. 180-191). Siena, Italia: CISCL Press.

Gómez Torrego, Leonardo. (2008). Cuestiones normativas en las estructuras del 
tipo Tener (Haber, Buscar, Encontrar)+X+Infinitivo. Español Actual, 89, 131135.

Grohmann, Kleanthes. (2003). Prolific domains: on the anti-locality of movement dependencies. Amsterdam, Holanda: John Benjamins.

Grohmann, Kleanthes, y Haegeman, Liliane. (2002). Resuming reflexives. Nordlyd, 31.3, 46-62.

Grohmann, Kleanthes and Panagiotidis, Phoevos (2005). An antilocality approach to Greek demonstratives. En Laura Brugè, Giuliana Giusti, Nicola Munaro, Walter Schweikert y Giuseppina Turano (Eds.), Contributions to the Thirtieth "Incontro di Grammatica Generativa" (pp. 243-263). Venice, Italia: Cafoscarina.

Grosu, Alexander. (1987). Pied-piping and the matching parameter. The Linguistic Review, 6, 41-58.

Grosu, Alexander. (1994). Three studies in locality and case. London, Inglaterra: Routledge.

Grosu, Alexander. (2004). The syntax-semantics of modal existential wh constructions. En Olga Mišeska Tomić (Ed.), Balkan syntax and semantics (pp. 405438). Amsterdam, Holanda: John Benjamins.

Grosu, Alexander y Landman, Fred. (1998). Strange relatives of the third kind. Natural Language Semantics, 6, 125-170.

Hernanz, M. Lluisa. (1999). El infinitivo. En Ignacio Bosque y Violeta Demonte (Eds.), Gramática descriptiva de la lengua española (pp. 2197-2356). Madrid, España: Espasa Calpe.

Hernanz, M. Lluisa. (2011). Sobre la periferia de los infinitivos. En M. Victoria Escandell, Manuel Leonetti y Cristina Sánchez (Eds.), 60 problemas de gramática (pp. 263-270). Madrid, España: Akal.

Hirschbühler, Paul y Rivero, María Luisa. (1981). A unified analysis of matching and non-matching free relatives in Catalan. En Victoria A. Burke y James Pustejovsky (Eds.), Proceedings of NELS 11 (pp. 113-124). Amherst, MA, Estados Unidos: GLSA Publications.

Izvorski, Roumyana. (1998). Non-indicative wh-complements of possessive and existential predicates. En Pius N. Tamanji \& Kiyomi Kusumoto (Eds.), Proceedings of NELS 28 (pp. 159-173). Amherst, MA., Estados Unidos: GLSA Publications.

Jiménez-Fernández, Ángel y Miyagawa, Shigeru. (2014). A feature-inheritance approach to root phenomena and parametric variation. Lingua, 145, 276-602.

Kempchinsky, Paula. (1986). Romance subjunctive clauses and Logical Form. Tesis Doctoral. California, Estados Unidos: UCLA.

Kempchinsky, Paula. (1990). Más sobre el efecto de referencia disjunta del subjuntivo, en Ignacio Bosque (Ed.), Indicativo y subjuntivo (pp. 234-258). Madrid, España: Taurus. 
Masullo, Pascual. (2011). ¿Lleva acento o no? Sobre un tipo de relativa libre poco estudiado. En M. Victoria Escandell, Manuel Leonetti y Cristina Sánchez (Eds.), 60 problemas de gramática (pp. 315-319). Madrid, España: Akal.

Milsark, Gary. (1974). Existential sentences in English. Tesis doctoral. Cambridge, MA, Estados Unidos: MIT.

Ojea, Ana. (2011). On mixed categories: the case of Free Relatives. SKY Journal of Linguistics, 24, 119-143.

Ojea, Ana. (2013). A uniform account of headless relatives in Spanish. Language Sciences, 40, 200-211.

Panagiotidis, Phoevos y Grohmann, Kleanthes. (2005). Mixed projections: categorial switches and prolific domains. Linguistic Analysis, 25, 141-161.

Pancheva-Izvorski, Roumyana. (2000). Free relatives and related matters. Tesis doctoral. Philadelphia, Estados Unidos: Universidad de Pennsylvania.

Partee, Barbara. (1976). Some transformational extensions of Montague grammar. En: Barbara Partee (Ed.), Montague Grammar (pp. 51-76). New York, Estados Unidos: Academic Press.

Pesetsky, David. (1982). Paths and categories. Tesis doctoral. Cambridge, MA, Estados Unidos: MIT.

Pesestsky, David y Torrego, Esther. (2007). The syntax of valuation and the interpretability of features. En Simin Karimi, Vida Samiia y Wendy K. Wilkins (Eds.), Phrasal and Clausal Architecture. Syntactic Derivation and Interpretation (pp. 262-294). Amsterdam, Holanda: John Benjamins.

Picallo, Carmen (1984). La interpretació obviativa i la noció "categoria de regim". Estudis Grammaticals 1, 217-248.

Plann, Susan. (1980). Relative clauses in Spanish without overt antecedents and related constructions. Berkely, Estados Unidos: University of California Press.

Quer, Josep. (2001). Interpreting mood. Probus, 13, 81-111.

RAE (Real Academia Española). (2009). Nueva gramática de la lengua española (Sintaxis II). Madrid, España: Espasa.

Ramsey, Marathon. (1894). A textbook of modern Spanish. New York, Estados Unidos: Holt, Rinehart and Wilson, 1965.

Rizzi, Luigi. (1997). The fine structure of the left periphery. En: Haegemann, Liliane (Ed.), Elements of Grammar (pp. 281-337). Dordrecht, Holanda: Kluwer.

Ross, John Robert. (1967). Constraints on Variables in Syntax. Tesis doctoral. Cambridge, MA, Estados Unidos: MIT.

Šimík, Radek. (2011) Modal existential wh-constructions. Tesis doctoral. Groningen, Holanda: Universidad de Groningen.

Smits, Rik. (1989). Eurogrammar. The relative and cleft constructions of the Germanic and Romance Languages. Dordrecht, Holanda: Foris.

Suñer, Margarita. (1984). Free Relatives and the Matching Parameter, The Linguistic Review, 3, 363-387. 
Táboas, Susana. (1995). Spanish infinitival relatives: A proposal about their indefiniteness requirement. Probus, 7, 197-219.

Ticio, M. Emma. (2003). On the structure of DPs. Tesis doctoral. Storrs, Estados Unidos: Universidad de Connecticut.

Ticio, M. Emma. (2006). Locality conditions in Spanish DPs. En Timothy L. Face and Carol A. Klee (Eds.), Selected Proceedings of the 8th Hispanic Linguistics Symposium (pp.137-153). Somerville, MA., Estados Unidos: Cascadilla Proceedings Project. 